\title{
Metazoarios parásitos de Micropterus salmoides (Perciformes: Centrarchidae) de reservorios de Nuevo León, México y su asociación con el factor de condición y sexo
}

\author{
Lucio Galaviz S., Baldemar Escobar G., Francisco Javier Iruegas B. \& Zinnia Judith Molina \\ Universidad Autónoma de Nuevo León, Facultad de Ciencias Biológicas, Unidad B. Ave. Universidad SN, Cd. \\ Universitaria, San Nicolás de los Garza, Nuevo León, 66455, México; lucio.galavizs1@uanl.edu.mx, \\ biolescobar@hotmail.com,firuegas@gmail.com,molinazinnia@hotmail.com
}

Recibido 03-VI-2015. Corregido 20-I-2016. Aceptado 25-II-2016.

\begin{abstract}
Metazoan parasites of Micropterus salmoides (Centrarchidae: Perciformes) from reservoirs of Nuevo León, México and their association with condition factor and gender. The largemouth bass Micropterus salmoides is a very valuable fish species for aquaculture and sport fishing; however, there are no systematic studies on fish metazoan parasites in Mexico. The main objective of the present study was to describe the prevalence, abundance, and intensity of $M$. salmoides metazoan parasites, and their association with fish condition factor and gender. The sample size was composed by 672 hosts, collected between 2011-2013 from the following reservoirs of Nuevo Leon, México: Rodrigo Gómez dam ("La Boca", LB), Cuchillo-Solidaridad dam (CS), Salinillas lagoon (LS), Mariano Escobedo dam ("Sombreretillo", S), and José López Portillo dam ("Cerro Prieto", CP). Living fish were transported to the laboratory; sizes and weights were then recorded to calculate the Fulton condition factor $(k)$. If possible, gender was also recorded. Parasites were detected under stereoscopy, recollected and preserved by traditional techniques. Statistical analysis of association between parasitic load, gender, and Fulton condition factor were calculated, using the $X^{2}$ and the Student- $t$ tests. Results showed that 12 different metazoans were identified, two flukes (Posthodiplostomum minimum centrarchi and Phyllodistomum pearsei), one tapeworm (Proteocephalus ambloplitis), three roundworms (Contracaecum sp., Spinitectus carolini and Philometra nodulosa), two acantocephalan (Neoechinorhynchus cylindratus and Arhythmorhynchus sp.), one leech (Myzobdella moorei), and three copepods (Ergasilus versicolor; Ergasilus arthrosis and Ergasilus cerastes). HSD Tukey test showed that infected fish from LB were significantly different than LS, CS, CP, and $\mathrm{S}(\mathrm{P}<0.05)$. Parasites most commonly collected in all five locations were P. m. centrarchi, Contracaecum sp. and E. versicolor. The frequency of $P . m$. centrarchi was highly significant $(\mathrm{P}<0.001)$ than other parasites; furthermore, this parasite showed the highest prevalence $(97.5 \%)$, abundance $(10.12-83.6)$, and intensity (15.44-88.5). Statistical dependence was found between intensity and abundance of metazoans with regard to female gender and size on five reservoirs $(t=143.82, \mathrm{P}=0.0001 ; t=2.6, \mathrm{P}=0.01$, respectively). In addition, the abundance and intensity showed statistical dependence with regard to $k$, which was proportionally significant. New locality records for Nuevo Leon include P. m. centrarchi, P. pearsei, P. ambloplitis, Contracaecum sp., Arhythmorhynchus sp., and E. cerastes. Furthermore, P. nodulosa, S. carolini, N. cylindratus, and E. arthrosis were new records for Mexico. Rev. Biol. Trop. 64 (2): 559-569. Epub 2016 June 01.
\end{abstract}

Key words: largemouth bass, helminth fauna, Ergasilus, Myzobdella, fish parasites.

La lobina o robalo (Micropterus salmoides Lacepède, 1802) es una especie muy apreciada en la acuacultura por su sabor y valor nutricional, por lo cual alcanza buena cotización en el mercado. Este pez también es un gran atractivo en las actividades turísticas como la pesca deportiva (Beltrán-Alvarez, Sánchez, Ramirez,
\& Ortega, 2013). El rango nativo de distribución de la lobina abarca desde Ontario, Canadá hasta el noreste de México, llegando a Chihuahua, Tamaulipas y Nuevo León (Brown, Runciman, Pollar, \& Grant, 2009; Lozano-Vilano, García, \& Espinosa, 2013; Salgado-Maldonado \& Rubio-Godoy, 2014). En el estado de Nuevo 
León se construyeron tres presas para almacenar agua de la Cuenca del Río Bravo y distribuirla a la zona metropolitana para uso residencial e industrial: Rodrigo Gómez (La Boca), Cuchillo Solidaridad y López Portillo (Cerro Prieto); también se encuentran dos lagunas naturales usadas para la agricultura y ganadería en los municipios rurales de Sabinas Hidalgo (Sombreretillo), y Anáhuac (Salinillas). En los cinco reservorios se realizan competencias de pesca de lobina como una de las actividades más importantes por la derrama económica en la región (INEGI, 2013).

En el área de estudio convergen tres diferentes provincias fisiográficas: a) La Llanura Costera de Norteamérica y b) la Llanura Costera del Golfo Norte, ambas ubicadas al norte y este central, respectivamente en la frontera con Tamaulipas) y c) la sierra Madre Oriental, al este y delimitando con el estado de Coahuila, lo que permite la existencia de una amplia biodiversidad y riqueza de especies acuáticas, sin embargo, son escasas las publicaciones oficiales sobre la helmintofauna en peces (Pérez-Ponce de León, G., Rosas-Valdez, R., Aguilar-Aguilar, R., Mendoza-Garfias, B., Mendoza-Palmero, ... Domínguez-Domínguez, 2010; Pérez-Ponce de León, García, León, \& Choudhury, 2000; Salgado-Maldonado, 2006) pues en la Cuenca del Rio Bravo ha sido reportado únicamente un estudio sobre metazoarios parásitos de M. salmoides (Aguilar-Aguilar, Martínez-Aquino, Espinosa-Pérez, \& Pérez-Ponce de León, 2014), en comparación con las numerosas contribuciones disponible en los Estados Unidos de Norteamerica (USA) donde un número substancial de casi todos los taxa (monogeneos, tremátodos, céstodos, nemátodos, acantocéfalos, copépodos) de parásitos han sido reportados (i.e. Lane, Spier, Wiederholt, \& Meagher, 2014; McGee, Whitney, \& Heckmann, 2001; Mizelle \& Crane, 1964; Richardson \& Abdo, 2011). Esta enorme diferencia dificulta el desarrollo de estrategias ecológicamente sustentables para implementar medidas profilácticas de control de enfermedades parasitarias que preserven este importante recurso. El presente estudio se realizó con los objetivos de determinar la prevalencia, abundancia e intensidad de los metazoarios parásitos que infectan a $M$. salmoides en los principales reservorios del estado y determinar aquellos metazoarios y su asociación estadística con el factor de condición y al sexo de los hospederos.

\section{MATERIALES Y MÉTODOS}

Área de estudio: Los peces fueron obtenidos de cinco reservorios localizados en el estado de Nuevo León, México (Los nombres comunes y abreviaciones se colocan entre paréntesis): Presa Rodrigo Gómez (comúnmente llamada "La Boca"; LB) y El CuchilloSolidaridad (CS); localizadas en los municipios de Santiago y China, respectivamente. Ambas fueron construidas en el afluente del Río San Juan. Laguna Salinillas, Anáhuac, NL (LS, surtida por el Río Salado) y la presa Mariano Escobedo (S, "Sombreretillo"), Sabinas Hidalgo. Estas cuatro se ubican en la cuenca del Río Bravo y la región hidrológica Bravo-Conchos. La presa José López Portillo (CP, "Cerro Prieto), ubicada en Linares, tiene dos ríos tributarios, el Hualahuises y el Pablillo, drenando en el Río Conchos. Esta es la única de las cinco que se encuentra en la región hidrológica San Fernando-Soto La Marina (la distribución de las localidades se muestra en la Fig. 1). Las coordenadas geográficas se citan en la cuadro 1 (INEGI, 2013).

Recolección de peces y examen helmintológico: En las localidades de estudio se obtuvieron 672 peces $M$. salmoides capturados mensualmente por los pescadores locales de agosto 2011 a septiembre 2013. Estos se mantuvieron en acuarios con aireadores conectados a una bomba de aire para llevarlos al laboratorio ubicado entre 80 y $267 \mathrm{~km}$ de las localidades y analizados a no más de $4 \mathrm{~h}$ después de capturarse. En el laboratorio se les tomaron las medidas (longitud, anchura) y se les identificó el sexo (Billings \& Munro, 1974). Primero se examinaron con el estereomicroscopio las aletas, cavidad oral y branquias para buscar ectoparásitos como copépodos o sanguijuelas. 


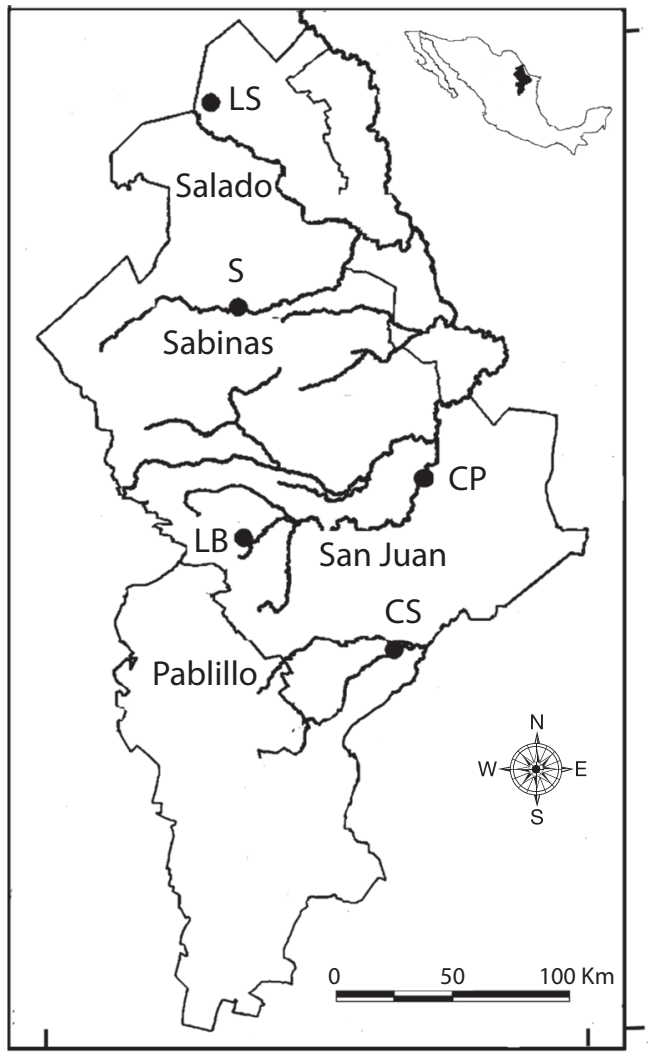

Fig. 1. Sitios de recolecta de Micropterus salmoides en el estado de Nuevo León, México. (LS) Salinillas, Anáhuac; (S) Sombreretillo, Sabinas, Hidalgo; (LB) Presa La Boca, Santiago; (CS) Presa Cuchillo-Solidaridad, China y (CP) Presa Cerro Prieto, Linares.

Fig. 1. Location of collection sites of largemouth bass Micropterus salmoides in Nuevo León state, Mexico. (LS) Salinillas, Anáhuac; (S) Sombreretillo, Sabinas, Hidalgo; (LB) Presa La Boca, Santiago; (CS) Presa CuchilloSolidaridad, China and (CP) Presa Cerro Prieto, Linares.

Después, las branquias fueron separadas en cajas de Petri, el tejido y la mucosa se rasparon con fórceps para detectar la posible presencia de organismos monogeneos. Posteriormente, se examinaron por separado los ojos, cerebro, músculo, hígado, bazo, riñón, corazón, gónadas, mesenterio y tracto gastrointestinal, y se colocó las muestras en cajas de Petri con solución salina fisiológica. Los parásitos se lavaron en solución salina limpia, se relajaron en formaldehído $4 \%$ caliente y se transfirieron a AFA (alcohol-formol-ácido acético) por

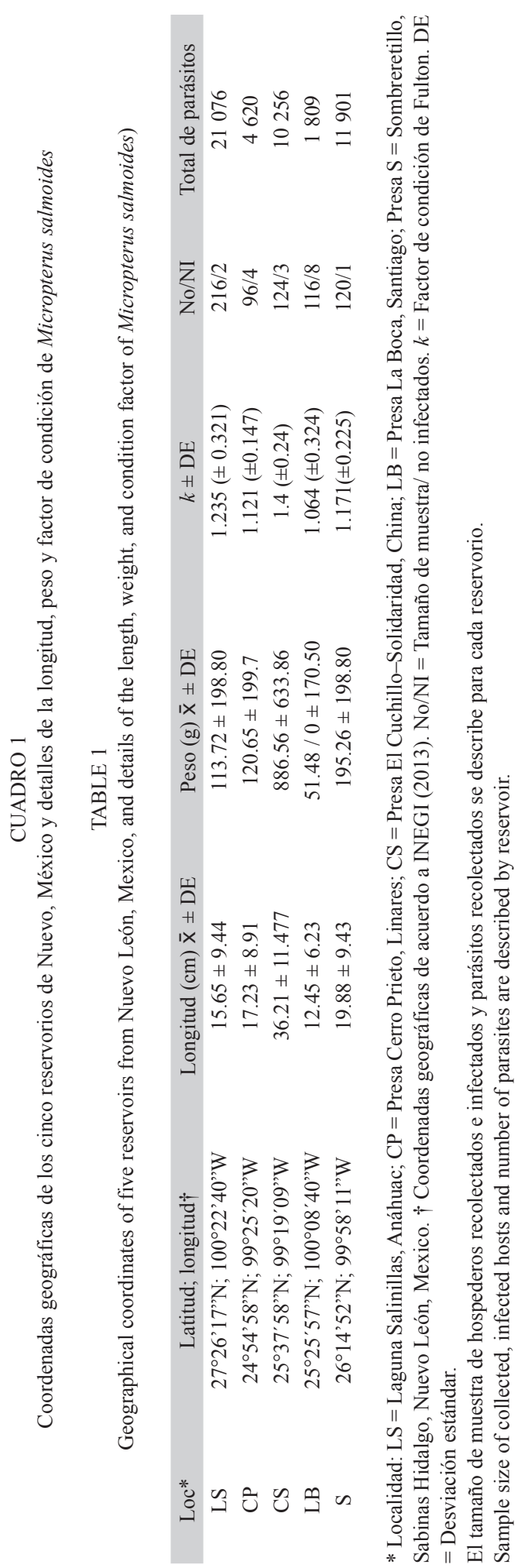


24-48 h, con excepción de los acantocéfalos, que se mantuvieron previamente por $12 \mathrm{~h}$ en agua destilada. Posteriormente, los platelmintos, acantocéfalos e hirudineos se lavaron en etanol $70 \%$ por $12-24 \mathrm{~h}$, y se tiñeron con hematoxilina de Van Cleave o Delafield, y se deshidrataron en una serie de etanoles, fueron transparentados en salicilato de metilo y montados con resina sintética (Entellan, Merck, Alemania). Los nemátodos fueron transferidos a etanol $70 \%$ y transparentados en lactofenol y analizados en preparaciones temporales. Todos los parásitos se estudiaron morfológicamente al microscopio y la morfometría se realizó con un objetivo micrométrico montado en un microscopio (Leica DM 750, Wetzlar, Alemania) según Vidal-Martínez, Aguirre-Macedo, Scholtz, González-Solís, y Mendoza (2002). Los helmintos fueron identificados hasta género según la taxa, con las claves de Yamaguti $(1958,1959,1961)$ mientras que la ubicación a especie se realizó con literatura especializada según cada caso.

Los especímenes de referencia se depositaron en la Colección de Helmintos de la UANL (CP: UANL) con los números de catálogo: Posthodiplostomum minimum centrarchi (MacCallum, 1921; Dubois, 1936) Hoffman, 1958 (UANL-HE 2 025); Phyllodistomum pearsei Holl, 1929 (UANL-HE 2 026); Proteocephalus ambloplitis (Leidy, 1887) Benedict, 1900 (UANL-HE 20 251); Contracaecum sp. (UANL-HE 20 251); Spinitectus carolini Holl, 1929 (UANL-HE 20 252); Philometra nodulosa Thomas, 1929 (UANL-HE 20 258); Neoechinorhynchus cylindratus Van Cleave, 1913 (UANL-HE 20 259); Arhythmorhynchus sp. (UANL-HE 20 259); Myzobdella moorei (Meyer, 1940) (UANL-HE 20 260); Ergasilus versicolor (Wilson 1911) (UANL-HE 20 261); Ergasilus arthrosis Roberts, 1969 (UANL-HE 20 262) y Ergasilus cerastes Roberts, 1969 (UANL-HE 20 263).

De cada hospedero se registró la longitud total, peso y el factor de condición de Fulton (Cuadro 1) con la formula $k=(\mathrm{W} / \mathrm{L} 3) \times 100$, donde $k$ es el factor de condición de Fulton, W es el peso y L la longitud total (Sarkar et al.,
2013), así como la desviación estándar (DE). El factor $k$ se comparó entre cada reservorio y con la carga parasitaria mediante análisis de varianza (ANOVA) seguida por la prueba de diferencias altamente significativas $(\mathrm{HSD}=$ highly significant difference test) de Tukey (Violante-González, Rojas-Herrera, \& AguirreMacedo, 2008). La prevalencia, intensidad media y abundancia media se calcularon de acuerdo a las definiciones dadas por Bush, Lafferty, Lotz \& Shostak (1977) y Valles-Ríos \& Ruiz-Campos (1996). Las comparaciones de las intensidades y abundancias de los parásitos y las diferencias significativas incluyeron análisis con la prueba $t$ de Student y la chi-cuadrada de Pearson $\left(X^{2}\right)$ con respecto al sexo y factor de condición entre infectados y no infectados cuando la prevalencia fue mayor al $5 \%$ con el programa SPSS.

\section{RESULTADOS}

Demografía de los hospederos: La longitud y peso (media $\pm \mathrm{DE}), k$ (media $\mathrm{y} \pm \mathrm{DE}$ ), distribución de muestras por localidad de los peces examinados, así como el número total de parásitos de los cinco reservorios, se muestran en el cuadro 1. Se obtuvieron diferencias significativas en la longitud, peso y $k$ (ANOVA, $F=$ 122.66, $\mathrm{P}=0.00001 ; F=136.76, \mathrm{P}=0.00001$; $F=27, \mathrm{P}=0.0001$, respectivamente) de las muestras de $M$. salmoides recolectadas en todas las localidades. Los hospederos de CS presentaron significativamente mayor peso, longitud y $k(\mathrm{P}=0.0001)$, mientras que los peces de $\mathrm{S}$ y LS presentaron los valores más bajos. El análisis con la prueba HSD de Tukey indicó que las longitudes y los pesos de las lobinas de CS eran significativamente mayores $(\mathrm{P}<0.05)$.

Las comparaciones entre intensidades y abundancias de parásitos con respecto al sexo y longitud muestra diferencias significativas entre las lobinas infectadas $(t=143.82, \mathrm{P}=$ $0.0001 ; t=2.6, \mathrm{P}=0.01$, respectivamente) $\mathrm{y}$ entre hembras y machos examinados en los cinco reservorios $(t=20.368, \mathrm{P}=0.0001)$. Al analizar la dependencia estadística entre la abundancia e intensidad con el factor de 
condición $k$, se observó que a mayor carga parasitaria aumenta la significancia; por ejemplo, en LS, donde se contabilizaron 18060 metacercarias de P. m. centrarchi, $\mathrm{P}=0.00001$ $(\mathrm{F}=7.93)$ con $X^{2}=50.13$ y $\mathrm{P}=0.001$; pero en LB, cuando la carga disminuye a 1174 la significancia aumenta al límite con valores de $\mathrm{P}=0.05$ (Cuadro 1). Igualmente, a niveles intermedios de carga, como se observó en CS con 9129 P. m. centrarchi, la significancia se ubicó en $\mathrm{P}=0.03$.

La prueba de Tukey HSD indicó que los peces infectados de LB fueron significativamente diferentes a los de LS, CS, CP y S (P $<0.05)$ debido a que este reservorio mostró la diversidad y abundancia más baja de parásitos (cinco diferentes especies con las abundancias más bajas y 1809 parásitos en total, Cuadros 1 y 2).

Parásitos: En total, 49662 especímenes de metazoarios fueron recolectados en $M$. salmoides analizados en los cinco reservorios of Nuevo León. El registro helmintológico establecido en este trabajo para M. salmoides, consta de 12 taxones: dos tremátodos digeneos, un céstodo, tres nemátodos, dos acantocéfalos, una sanguijuela y tres copépodos.

En las 672 lobinas examinadas, 18 no estaban parasitadas, del resto se recolectó al menos una especie de metazoario parásito (Cuadro 1). La mayoría de los peces (97.31\%) hospedaban de 2 a 4 parásitos. Los parásitos que se recolectaron más comúnmente en las cinco localidades fueron Posthodiplostomum minimum centrarchi, Contracaecum sp., y Ergasilus versicolor y su presencia fue altamente significativa $\left(X^{2}=\right.$ 7 646.8, $\mathrm{P}=0.0001)$ en las localidades. La recolección de P.m. centrarchi en los cinco reservorios fue la más significativa (ANOVA, $F=17.61, \mathrm{P}<0.001)$, además presentó la más elevada prevalencia (97.5\%), abundancia (10.12-83.6) e intensidad (15.44-88.5), como se muestra en el cuadro 2; la prueba de Tukey reveló que la abundancia de P.m. centrarchi en LS fueron significativamente más altas que en $\mathrm{S}(\mathrm{P}<0.05)$. Myzobdella moorei, Neoechinorhynchus cylindratus, y Ergasilus versicolor, presentaron las abundancias más bajas considerando a los que distribuyeron en cuatro o más localidades $(\mathrm{HSD}=0.031-7.9 ; \mathrm{HSD}=0.241$ $7.02, \mathrm{y} \mathrm{HSD}=0.01-11.95$, respectivamente).

\section{DISCUSIÓN}

En la presa CS los peces tuvieron mayor factor de condición con una carga de parásitos moderada, en comparación con LS con la mayor, o LB con la menor carga (21 076 y 1 809 , respectivamente). Las otras cuatro localidades, al igual que la significancia estadística, a diferencia de S y LS donde la condición física y talla es menor y la carga de parásitos aumenta hasta casi el doble (21 076). Así de igual forma, se observó que también varía la significancia de manera proporcional (Wilson \& Camp, 2003).

También se presentó dependencia estadística entre la talla y nivel de infección en los cinco reservorios, predominando las mayores parasitosis en las hembras y con tallas mayores a $20 \mathrm{~cm}$ (358). La misma interrelación estadística se ha comprobado en Micropterus dolomieu y M. salmoides del Gull Lake, Michigan, así como también Lepomis macrochirus de Indiana, parasitados con $P$. ambloplitis, $P$. $m$. centrarchi y $S$. carolini, quienes presentan la misma interrelación en el incremento en la intensidad y abundancia con la longitud (Gillilland \& Muzzall, 2004; Wilson \& Camp, 2003) debido a que los peces más grandes ingieren mayor cantidad de alimento (Amin \& Cowen, 1990). Sobre la significancia entre la parasitosis y el sexo, resultados similares se han reportado en Contracaecum y Polyacanthorhynchus kenyensis en hembras de Micropterus salmoides de lagos de Kenya (Aloo, 1999).

Así, aunque la lobina M. salmoides tiene una gran importancia económica, no existen estudios sistemáticos oficiales sobre los helmintos parásitos de este hospedero en México. Entre los escasos reportes se encuentran los realizados en Durango, que es la zona más cercana donde se han analizado ejemplares de $M$. salmoides $(\mathrm{n}=8)$ entre otras 17 especies de peces, describiéndose a Centrocestus 


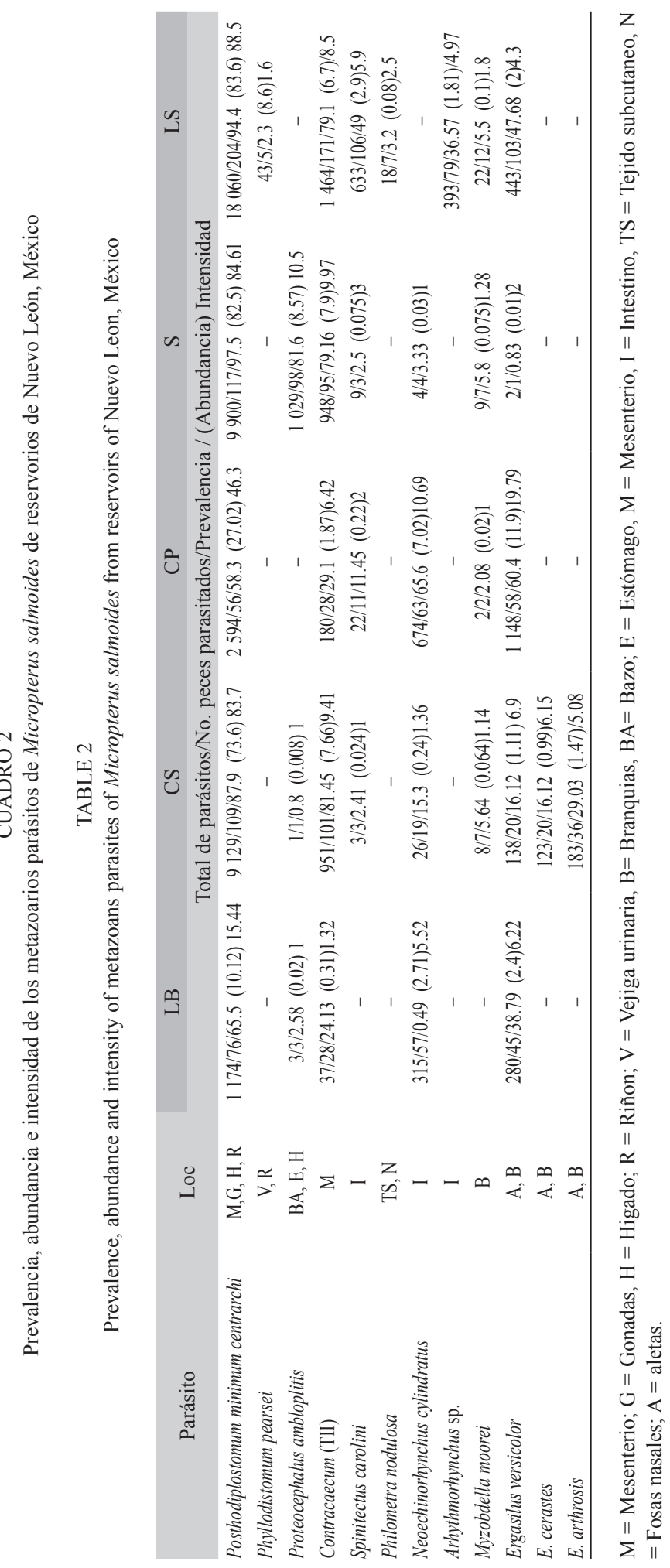


formosanus (Nishigori, 1924), Serpinema trispinosum (Leidy, 1852), Spinitectus sp., Spiroxis $\mathrm{sp}$., y Leptorhynchoides thecatus Linton, 1891 (Aguilar-Aguilar, et al., 2014). SalgadoMaldonado (2006) reportó a Bothriocephalus acheilognathi Yamaguti, 1934 de Guanajuato. Pérez-Ponce de León et al. (2000) estudiaron ejemplares de M. salmoides del Lago de Pátzcuaro, Michoacán y describen a Crepidostomum cornutum (Osborn, 1903); Clinostomum complanatum (Rudolphi, 1814) y Diplostomum sp., lo cual difiere a la helmintofauna descrita en $M$. salmoides de Nuevo León.

Posthodiplostomum minimum centrarchi se ha recolectado en México de M. salmoides, Lepomis macrochirus, L. megalotis, Pomoxis annularis, Chirostoma jordani, Alloophorus robustus, Goodea atripinnis y Xenotoca variata del Lago de Pátzcuaro y Lago de Cuitzeo, Michoacan, Río Nazas, Durango, México (descrito como P. minimum por Guzmán-Cornejo \& García-Prieto, 1999; Pérez-Ponce de León et al., 2000, 2010). Este es nuevo registro para Nuevo León, extiende su rango geográfico (desde Michoacán y Durango) donde P. m. centrarchi ha sido reportado.

Este trabajo se considera también como un nuevo registro de localidad para México de Phyllodistomum pearsei $(=$ P. superbum $)$, pues a la fecha solo ha sido recolectado de Enneacanthus glorius en Estados Unidos (Carolina del Norte), Centrarchus macropterus, Lepomis gibbosus, L. macrochirus, L. gulosus y Pomoxis annularis (i. e. Howard \& Aliff, 1980; McAllister \& Bursey, 2013). El sitio más cercano donde se ha recolectado es en $M$. salmoides) de Texas (Ingham \& Dronen, 2004), donde coinciden las elevadas prevalencias e intensidades de $P$. minimum $(77.8,93 \%$, respectivamente) y moderada a baja en Clinostomum marginatum (50, 4.2\%), P. ambloplitis (27.8, $6.4 \%)$, S. carolini (16.6, 6-7 \%), P. nodulosa (33, $8 \%)$, Contracaecum sp. $(39.8,4 \%)$ y $N$. cylindratus (22.2, $2.2 \%)$.

En México, los plerocercoides de Proteocephalus ambloplitis han sido recolectados de Centrarchidae (M. salmoides, Lepomis macrochirus); Atherinopsidae (Chirostoma jordani);
Cyprinidae (Yuriria alta), Goodeidae (Goodea atripinnis, Xenotoca variata) de Guanajuato, México (Salgado-Maldonado, 2006; SalgadoMaldonado \& Rubio-Godoy, 2014). También se ha recolectado de Catastomidae, Centrarchidae, Ictaluridae, Lepososteidae y Percidae en Wisconsin, USA (Amin, 1990; Gillilland \& Muzzall, 2004; Muzzall \& Whelan, 2011). Esta es la primera vez que se recolecta en peces de Nuevo León.

Contracaecum sp. ha sido descrito en 18 familias y 67 especies de peces de casi todos los estados mexicanos (Salgado-Maldonado, 2006). Contracaecum ha sido reportado también en $M$. salmoides de la presa Marte R. Gómez, Tamaulipas (Pérez-Ponce de León, Mendoza-Garfias, Rosas-Valdez, \& Choudhury, 2013), por lo cual, el presente registro representa un nuevo reporte de localidad para Nuevo León. Spinitectus carolini no ha sido reportado en el país, diferenciándose de las especies descritas en peces de México ( $S$. osorioi Choudhury \& Pérez-Ponce de León, 2001, específico de los hospederos Chirostoma attenuatum y C. estor del Lago de Pátzcuaro, Michoacan, México y $S$. mexicanus CaspetaMandujano, Moravec, \& Salgado-Maldonado, 2000, de los peces dulceacuícolas Heterandria bimaculata) en la posición del poro excretor entre la hilera de espinas 8-9 y carecer de la protuberancia distal distintiva de la espícula izquierda (Caspeta-Mandujano, et al., 2000; Choudhury \& Pérez-Ponce de León, 2001). Spinitectus carolini es muy frecuente y parasita un amplio rango de peces de Estados Unidos incluyendo centrárquidos (Bangham, 1955; Bauer \& Whipps, 2013; Fellis \& Esch, 2004; Steinauer \& Font, 2003). Por estos motivos, en el presente trabajo se presenta un nuevo registro de $S$. carolini parasitando M. salmoides para México. Igualmente, sobre Philometra nodulo$s a$ no existen reportes en México, solo de algunos estados de Estados Unidos en Catastomus commersoni de Michigan y Wyoming (Clifford \& Facciani, 1972; Thomas, 1929) así como de Carpioides velifer de Indiana (Buckner \& Buckner, 1985) y M. salmoides de Texas (Meade \& Bedinger, 1972) por lo cual el presente 
constituye un nuevo reporte de localidad para México. El acantocéfalo Neoechinorhynchus cylindratus se recolectó por primera vez en $M$. salmoides analizados en México; anteriormente se ha reportado de algunos estados de Estados Unidos (i.e. Aliff, Smith, \& Lucas, 1977; Muzzall \& Gillilland, 2004; Richardson \& Abdo, 2011), pero nuestro reporte es el primero para México. El hirudineo M. moorei se ha reportado de carpa y bagre de Tamaulipas y Nuevo León (Galaviz-Silva, Witt-Sepulveda

Mercado-Hernández, \& Martínez-Hernández, 1990). Sobre el género Arhythmorhynchus Lühe 1911 solo ha sido reportado a $A$. brevis Van Cleave, 1916 en M. salmoides y en Algansea lacustris (acumara) del Lago de Pátzcuaro (Pérez-Ponce de León, Garcia, Osorio, \& León-Règagnon, 1996), por lo cual esta constituye la primera vez que se recolecta en Nuevo León, México.

Ergasilus versicolor ha sido reportado de Mugil cephalus Linnaeus, 1758 de del Río Colorado, Baja California Sur (Valles-Ríos, Ruiz-Campos, \& Galaviz-Silva, 2000) y de bagre Ictalurus punctatus; Galaviz-Silva et al. (1990) esta es la primera vez que se reporta de $M$. salmoides. Sobre E. arthrosis esta es la primer vez que se recolecta en peces de México por lo cual es el primer reporte de localidad y de hospedero. Ergasilus cerastes en cambio, se ha reportado de I. punctatus de granjas de Tamaulipas (Rodríguez-Almaraz, Gómez-Flores, \& Rábago-Castro, 2012) y el presente extiende la distribución geográfica hasta Nuevo León.

En conclusión, este trabajo describe la significancia estadística entre talla, peso y factor de condición de la lobina y demuestra que las parasitosis causadas por P. m. centrarchi y Contracaecum sp., afectan el factor de condición de acuerdo a la carga de parásitos lo cual es un factor de riesgo para el desarrollo sustentable de este importante recurso pesquero. Estos resultados abren el camino a estrategias ecológicamente sustentables para implementar medidas profilácticas de control en ecosistemas silvestres, como ejemplo, métodos mixtos de control químico y biológico, que han probado tener una eficiencia del 95-99\% (Noatcha \& Whitledge, 2011). También, este es el primer estudio de la fauna endoparasitaria de helmintos, así como de ectoparásitos copépodos y sanguijuelas de $M$. salmoides para Nuevo León, realizado en forma sistemática en México y particularmente en la cuenca del Río Bravo y región hidrológica Bravo-Conchos.

\section{AGRADECIMIENTOS}

Este estudio se efectuó con el proyecto No. CN-835-11 (PAICYT-UANL) "Parasitofauna de peces silvestres y cultivados en la región hidrológica Bravo-Conchos del estado de Nuevo León, México y análisis filogenético molecular de helmintos de importancia en salud pública". Agradecemos especialmente a Hesperia Andrea Corzo Ariyama y Jesús Dávila Barboza por colaborar en la necropsia y recolección de parásitos.

\section{RESUMEN}

Micropterus salmoides es una especie muy apreciada en la acuacultura y pesca deportiva, sin embargo, no existen estudios sistemáticos sobre los parásitos en México. El objetivo principal del presente estudio fue describir la prevalencia, abundancia e intensidad de los metazoarios parásitos de $M$. salmoides y su asociación con el factor de condición y el sexo. El tamaño de muestra fue de 672 hospederos, recolectados entre 2011-2013 de los siguientes reservorios de Nuevo León, México: Presa Rodrigo Gómez ("La Boca", LB); Presa Cuchillo-Solidaridad (CS), Laguna Salinillas (LS), Presa Mariano Escobedo ("Sombreretillo", S), y Presa José López Portillo ("Cerro Prieto", CP). Los peces fueron transportados vivos al laboratorio, se les registró el tamaño y peso para calcular el coeficiente de condición de Fulton $(k)$. El sexo también se registró cuando fue posible. Los parásitos fueron detectados con el estereoscopio, recolectados y preservados con las técnicas parasitológicas tradicionales. Se calcularon los análisis estadísticos de asociación entre la carga parasitaria, sexo y factor de condición usando $X^{2}$ y la prueba de $t$ de Student. Los resultados mostraron la identificación de 12 metazoarios diferentes: dos tremátodos (Posthodiplostomum minimum centrarchi y Phyllodistomum pearsei); un céstodo (Proteocephalus ambloplitis); tres nemátodos (Contracaecum sp.; Spinitectus carolini y Philometra nodulosa); dos acantocéfalos (Neoechinorhynchus cylindratus y Arhythmorhynchus sp.); un hirudineo (Myzobdella moorei) y tres copépodos (Ergasilus versicolor; Ergasilus 
arthrosis y Ergasilus cerastes). La prueba de Tukey HSD mostró que los peces infectados de LB fueron significativamente diferentes a los de LS, CS, CP y S $(\mathrm{P}<0.05)$. Los parásitos que se recolectaron más comúnmente en las cinco localidades fueron: P. m. centrarchi, Contracaecum sp., y E. versicolor. La frecuencia de $P$. $m$. centrarchi fue altamente significativa $(\mathrm{P}<0.001)$ en comparación con los otros parásitos; además presentó la más elevada prevalencia (97.5\%), abundancia (10.12-83.6) e intensidad (15.4488.5). Se encontró asociación estadística entre la intensidad y abundancia de los metazoarios con respecto al sexo femenino y tamaño en los cinco reservorios $(t=143.82$, P $=0.0001 ; t=2.6, \mathrm{P}=0.01$, respectivamente). Además, la abundancia e intensidad mostraron asociación estadística con respecto a $k$, lo cual fue proporcionalmente significativo. Se incluyen nuevos registros de localidad para $P$. $m$. centrarchi, P. pearsei, P. ambloplitis, Contracaecum sp., Arhythmorhynchus sp. y E. cerastes. Además, P. nodulosa, S. carolini, N. cylindratus, y E. arthrosis se consideran como nuevos registros para México.

Palabras clave: Lobina, helmintofauna, Ergasilus, Myzobdella, parásitos de peces.

\section{REFERENCIAS}

Aguilar-Aguilar, R., Martínez-Aquino, A., Espinosa-Pérez, H., \& Pérez-Ponce de León, G. (2014). Helminth parasites of freshwater fishes from Cuatro Ciénegas, Coahuila, in the Chihuahuan desert of Mexico: inventory and biogeographical implications. Integrative Zoology, 9, 328-339.

Aliff, J. V., Smith, D., \& Lucas, H. (1977). Some metazoan parasites from fishes of Middle Georgia. Transactions of the American Microscopical Society, 96, 145-148.

Aloo, P. A. (1999). Ecological studies of helminth parasites of the largemouth bass, Micropterus salmoides, from Lake Naivasha and the Oloidien Bay, Kenya. The Onderstepoort Journal of Veterinary Research, 66, 73-79.

Amin, O. M. (1990). Cestoda from lake fishes in Wisconsin: occurrence of Proteocephalus in Esox and other fish species. Proceedings of the Helminthological Society of Washington, 57, 132-139.

Amin, O. M., \& Cowen, M. (1990). Cestoda from Lake Fishes in Wisconsin: The ecology of Proteocephalus ambloplitis and Haplobothrium globuliforme in bass and bowfin. Journal of the Helminthological Society of Washington, 57, 120-131.

Bangham, R. V. (1955). Studies on fish parasites of Lake Huron and Manitoulin Island. American Midland Naturalist, 53, 184-194.
Bauer, E. F., \& Whipps, C. M. (2013). Parasites of two native fishes in adjacent Adirondack lakes. Journal of Parasitology, 99, 603-609.

Beltrán-Alvarez, R., Sánchez Palacios, J., Ramírez Lozano, J. P., \& Ortega Salas, A. A. (2013). Reproducción de Micropterus salmoides (Pisces: Centrarchidae), en el embalse Gustavo Díaz Ordaz, Sinaloa, México. Revista de Biología Tropical, 61, 1313-1325.

Billings, C., \& Munro, J. (1974). The biology, ecology and bionomics of Caribbean reef fishes: Pomadasydae (Grunts). Part 5. Research Report from the Zoology Department, University of the West Indies, 3, 1-128.

Brown, T. G., Runciman, B., Pollard, S., \& Grant, D. A. (2009). Biological synopsis of largemouth bass (Micropterus salmoides). Canadian Manuscript Report of Fisheries and Aquatic Sciences, 2884, 1-26.

Buckner, R. L., \& Buckner, S. C. (1985). Parasitic endohelminths from fishes of Southern Indiana. Indiana Academy of Science, 94, 615-620.

Bush, A. O., Lafferty, K. D., Lotz, J. M., \& Shostak, A. W. (1997). Parasitology meets ecology on its own terms: Margolis et al. revisited. Journal of Parasitology, 83, 575-583.

Caspeta-Mandujano, J. M., Moravec, F., \& SalgadoMaldonado, G. (2000). Spinitectus mexicanus n. sp. (Nematoda: Cystidicolidae) from the intestine of the freshwater fish Heterandria bimaculata in México. Journal of Parasitology, 86, 83-88.

Choudhury, A., \& Pérez-Ponce de León, G. (2001). Spinitectus osorioi n. sp. (Nematoda: Cystidicolidae) from Chirostoma spp. (Osteichthyes: Atherinidae) in Lake Pátzcuaro, Michoacán, México. Journal of Parasitology, $87,648-655$

Clifford, T. J., \& Facciani, S. (1972). Philometra nodulosa in Wyoming white suckers. The Progressive FishCulturist, 34, 235-236.

Fellis, K. J., \& Esch, G. W. (2004). Community structure and seasonal dynamics of helminth parasites in Lepomis cyanellus and L. macrochirus from Charlie's Pond, North Carolina: Host size and species as determinants of community structure. Journal of Parasitology, 90, 41-49.

Galaviz-Silva, L., Witt-Sepulveda, G. D., Mercado-Hernández, R., \& Martínez-Hernández, J. J. (1990). New localities for monogenic trematodes and other ectoparasites of carp Cyprinus carpio and catfish Ictalurus punctatus in northeastern Mexico and their relations with some biotic and abiotic factors. Journal of the Elisha Mitchell Scientific Society, 106, 64-77.

Gillilland III, M. G., \& Muzzall, P. (2004). Microhabitat analysis of bass tapeworm, Proteocephalus ambloplitis (Eucestoda: Proteocephalidae), in smallmouth bass, Micropterus dolomieu, and largemouth bass, 
Micropterus salmoides, from Gull Lake, Michigan, U.S.A. Comparative Parasitology, 71, 221-225.

Guzmán-Cornejo, M. C. \& García-Prieto, L. (1999). Trematodiasis en algunos peces del lago de Cuitzeo, Michoacán, México. Revista de Biología Tropical, 47, 593-596

Howard, C. N., \& Aliff, J. V. (1980). Metazoan parasites of fishes from Piedmont and coastal plain Georgia. Georgia Journal of Science, 8, 173-179.

INEGI (Instituto Nacional de Estadística y Geografía). (2013). Conociendo Nuevo León. Recuperado de http://www.inegi.org.mx/prod_serv/ contenidos/espanol/bvinegi/productos/estudios/conociendo/NUEVO_LEON.pdf

Ingham, R. E., \& Dronen, N. O. (2004). Some effects of seasonality on helminthic infection in largemouth bass, Micropterus salmoides (Lacepede) (Centrarchidae). The Southwestern Naturalist, 27, 223-225.

Lane, B., Spier, T., Wiederholt, J., \& Meagher, S. (2014). Host Specificity of a parasitic fluke: Is Posthodiplostomum minimum a centrarchid-infecting generalist or specialist? Journal of Parasitology, 101, 6-17.

Lozano-Vilano, M. L., García-Ramírez, M. E., \& EspinosaNarváez, M. A. (2013). Peces. En C. Cantú-Ayala, M. Rovalo-Merino, \& J. Marmolejo (Eds.), Historia Natural del Parque Nacional Cumbres de Monterrey, México (pp. 195-206). Nuevo León, México: Dirección General de Publicaciones, Universidad Autónoma de Nuevo León.

McAllister, C. T., \& Bursey, C. R. (2013). Noteworthy trematode (Digenea) parasites of the pirate perch, Apherododerus sayanus (Percopsiformes: Aphredoderidae), from Southeastern Oklahoma. Proceedings of the Oklahoma Academy of Science, 93, 37-40.

McGee, M. N., Whitney, M. J., \& Heckmann, R. A. (2001). The monogenean Haplocleidus furcatus Mueller, 1937 (Phylum Platyhelminthes) on Lepomis cyanellus Rafinesque, 1819 from Utah: A range extension. Western North American Naturalist, 61, 245-247.

Meade, T. G., \& Bedinger, Jr., C. A. (1972). Helminth parasites in some species of fresh water fishes of Eastern Texas. Southwestern Naturalist, 16, 281-295.

Mizelle, J. D., \& Crane, J. W. (1964). Studies on monogenetic trematodes. XXIII. Gill parasites of Micropterus salmoides (Lacépéde) from a California pond. Transactions of the American Microscopical Society, $83,343-348$

Muzzall, P. M., \&. Gillilland, III, M. G. (2004). Occurrence of acanthocephalans in largemouth bass and smallmouth bass (Centrarchidae) from Gull Lake, Michigan. Journal of Parasitology, 90, 663-664.
Muzzall, P. M., \& Whelan, G. (2011). Parasites from the Great Lakes: A synopsis and review of the literature, 1871-2010. Great Lakes Fisheries Commision. Ann Arbor: Miscellaneous Publication 2011-01.

Noatcha, M. R., \& Whitledge, G. W. (2011). An evaluation of hydrated lime and predator sunfish as a combined chemical-biological approach for controlling snails in aquaculture ponds. North American Journal of Aquaculture, 73, 53-59.

Pérez-Ponce de León, G., Garcia, L., Osorio, D., \& LeónRègagnon, A. N. D. (1996). Listados faunísticos de México. VI Helmintos Parásitos de peces de aguas continentales de México. Serie Listados Faunísticos. Distrito Federal, México: Instituto de Biología Universidad Nacional Autónoma de México.

Pérez-Ponce de León, G., García-Prieto, L., León-Règagnon, V., \& Choudhury, A. (2000). Helminth communities of native and introduced fishes in Lake Pátzcuaro, Michoacán, México. Journal of Fish Biology, 57, 303-325.

Pérez-Ponce de León, G., Mendoza-Garfias, B., RosasValdez, R., \& Choudhury, A. (2013). New host and locality records of freshwater fish helminth parasites in river basins north of the Transmexican Volcanic Belt: another look at biogeographical patterns. Revista Mexicana de Biodiversidad, 84, 556-562.

Pérez-Ponce de León, G., Rosas-Valdez, R., Aguilar-Aguilar, R., Mendoza-Garfias, B., Mendoza-Palmero, C., García-Prieto, L., Rojas-Sánchez, A., Briosio-Aguilar, R., Pérez-Rodríguez, R., \& Domínguez-Domínguez, O. (2010). Helminth parasites of freshwater fishes, Nazas River basin, northern Mexico. Check List, 6, 26-35.

Richardson, D. J., \& Abdo, A. (2011). Postcyclic transmission of Leptorhynchoides thecatus and Neoechinorhynchus cylindratus (Acanthocephala) to largemouth bass (Micropterus salmoides). Comparative Parasitology, 78, 233-235.

Rodríguez-Almaraz, G. A., Gómez-Flores, R., \& RábagoCastro, J. (2012). First records of Ergasilus cerastes (Copepoda, Poecilostomatoida) on cage-reared Ictalurus from Northeast Mexico. Crustaceana, 85, 607-615.

Salgado-Maldonado, G. (2006). Checklist of helminth parasites of freshwater fishes from Mexico. Zootaxa, $1324,1-357$

Salgado-Maldonado, G., \& Rubio-Godoy M. (2014). Helmintos parásitos de peces de agua dulce introducidos. En R. Mendoza, \& P. Koleff (Eds.), Especies acuáticas invasoras en México (pp. 269-285). México: Comisión Nacional para el Conocimiento y Uso de la Biodiversidad.

Sarkar, U. K., Khan, G. E., Dabas, A., Pathak, A. K., Mir, J. I., Rebello, S. C., \& Singh, P. S. (2013). Length 
weight relationship and condition factor of selected freshwater fish species in River Ganga, Gomti, and Rapti, India. Journal of Environmental Biology, 34, 951-956.

Steinauer, M. L., \& Font, W. F. (2003). Seasonal dynamics of the helminths of bluegill (Lepomis macrochirus) in a subtropical region. Journal of Parasitology, 89, 324-328.

Thomas, L. J. (1929). Philometra nodulosa nov. spec.: With Notes on the Life History. Journal of Parasitology, 15, 193-198.

Valles-Ríos, M. E. \& Ruiz-Campos, G. (1996). Prevalencia e intensidad de helmintos parásitos del tracto digestivo de la trucha arcoiris Onchorhynchus mykiss nelsoni (Pisces: Salmonidae) de Baja California, México. Revista de Biología Tropical, 44, 579-584.

Valles-Ríos, M. E., Ruiz-Campos, G., \& Galaviz-Silva, L. (2000). Parasite prevalence and intensity in Mugil cephalus (Pisces: Mugilidae), from Colorado River, Baja California, Mexico. Revista de Biología Tropical, 48, 495-501.
Vidal-Martínez, V., Aguirre-Macedo, M. L., Scholtz, T., González-Solís, D., \& Mendoza, E. F. (2002). Atlas de los helmintos parásitos de cíclidos en México. Yucatán México: Dirección General de Publicaciones, Instituto Politécnico Nacional.

Violante-González, J., Rojas-Herrera, A., \& Aguirre-Macedo, M. L. (2008). Seasonal patterns in metazoan parasite community of the "Fat Sleeper" Dormitator latifrons (Pisces: Eleotridae) from Tres Palos Lagoon, Guerrero, Mexico. Revista de Biología Tropical, 56, 1419-1427.

Wilson, S., \& Camp, J. W. (2003). Helminths of bluegills, Lepomis macrochirus, from a Northern Indiana Pond. Comparative Parasitology, 70, 88-92.

Yamaguti, S. (1958). The Digenetic Trematodes of Vertebrates. Systema Helminthum. Volume I. (Part I). New York: Interscience Publications.

Yamaguti, S. (1959). Systema Helminthum. Vol II. The Cestodes of Vertebrates. New York: Interscience Publications.

Yamaguti, S. (1961). Systema Helminthum. Vol III. Nematodes. New York: Interscience Publications. 
